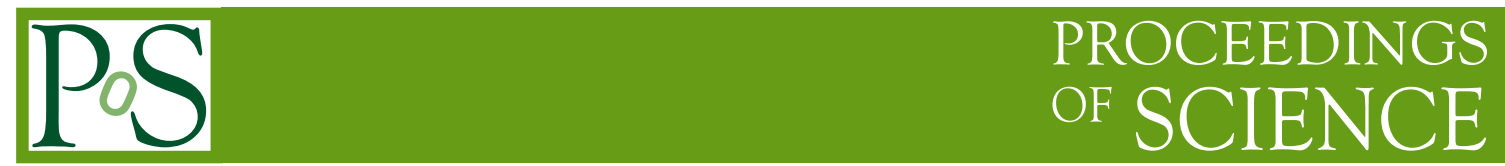

\title{
Review of DVCS results at HERMES
}

\section{Hrachya Marukyan*i}

Yerevan Physics Institute, Alikhanian Brs. St. 2, Yerevan, Armenia

E-mail: marukyan@mail.desy.de

\begin{abstract}
We report an overview of results for the deeply virtual Compton scattering process measured with the HERMES experiment at DESY, Hamburg. These measurements are exploiting the rich data set collected with the longitudinally polarized $27.6 \mathrm{GeV}$ HERA lepton beams of both charges and spin orientations scattered off longitudinally or transversely polarized or unpolarized gas targets (H, D or heavier nuclei). The Fourier amplitudes of various azimuthal asymmetries are extracted from this data. These amplitudes are sensitive to the imaginary or real part of certain Compton form factors, therefore generalized parton distributions, or their linear combinations.
\end{abstract}

XVIII International Workshop on Deep-Inelastic Scattering and Related Subjects, DIS 2010 April 19-23, 2010

Firenze, Italy

\footnotetext{
*Speaker.

$\dagger$ On behalf of the HERMES Collaboration
} 


\section{Introduction}

The interest in hard exclusive processes over the last decade has its origin in the theoretical description of these processes in terms of the Generalized Parton Distribution (GPD) formalism [1]. The GPD formalism incorporates the well-known one-dimensional nucleon form factors and (transverse-momentum-integrated) parton momentum distribution functions in a multidimensional picture of the nucleon, and correlates these transverse spacial and longitudinal momentum distributions of partons. Interest in the GPD framework has been intensified by the fact that the unknown total angular momentum components of the quarks and gluons are encoded in the GPDs [2].

\section{DVCS measurements at HERMES}

One of the theoretically cleanest channels to probe GPDs is deeply virtual Compton scattering (DVCS) in which a single real photon is produced in the final state leaving the recoiling nucleon (nucleus) intact. The spin-1/2 nucleon, is described by four leading-twist quark-chiralityconserving GPDs $H, E, \widetilde{H}$ and $\widetilde{E}$ [3]. In contrast, DVCS leaving the spin-1 deuteron intact requires nine GPDs- $H_{1}, H_{2}, H_{3}, H_{4}, H_{5}, \widetilde{H}_{1}, \widetilde{H}_{2}, \widetilde{H}_{3}$ and $\widetilde{H}_{4}-$ to describe all observables [4]. Measurement of the DVCS process is intrinsically mixed with that of the Bethe-Heitler $(\mathrm{BH})$ process in which a real photon is radiated by the lepton. The cross section for the leptoproduction of real photons is therefore proportional to the square of the sum of both amplitudes [4]

$$
\frac{\mathrm{d} \sigma}{\mathrm{d} x_{A} \mathrm{~d} Q^{2} \mathrm{~d}|t| \mathrm{d} \phi}=\frac{x_{A} y^{2}}{32(2 \pi)^{4} Q^{4}} \frac{\left|\tau_{B H}\right|^{2}+\left|\tau_{D V C S}\right|^{2}+\tau_{B H}^{*} \tau_{D V C S}+\tau_{D V C S}^{*} \tau_{B H}}{\sqrt{1+\varepsilon^{2}}}
$$

where $-Q^{2}$ represents the virtual photon four-momentum squared, $y$ the fraction of the incident lepton energy carried by the virtual photon in the target rest frame, $t$ the Mandelstam variable, $x_{A}$ is related to the Bjorken scaling variable and $\varepsilon \equiv 2 x_{A} M_{A} / Q$. The azimuthal angle $\phi$ is defined as the angle between the lepton scattering plane and the photon production plane spanned by the virtual and real photons.

The cross section for a longitudinally polarized lepton beam scattered off an unpolarized hydrogen or deuterium target can be expressed as

$$
\sigma_{L U}\left(P_{l}, e_{l}, \phi\right)=\sigma_{U U}(\phi) \cdot\left\{1+P_{l} \mathrm{~A}_{\mathrm{LU}}^{\mathrm{DVCS}}(\phi)+e_{l} \mathrm{~A}_{\mathrm{CU}}(\phi)+e_{l} P_{l} \mathrm{~A}_{\mathrm{LU}}^{\mathrm{I}}(\phi)\right\},
$$

where $e_{l}$ and $P_{l}$ are the lepton beam charge and polarization. The beam-helicity asymmetries (BSAs) $\mathrm{A}_{\mathrm{LU}}^{\mathrm{DVCS}}$ and $\mathrm{A}_{\mathrm{LU}}^{\mathrm{I}}$ are related to certain Compton form factors (CFFs) in a Fourier series of expansion in $\phi$ of the pure DVCS squared and interference term, respectively. The beam-charge asymmetry (BCA) $A_{C U}$ is related only to the even Fourier harmonics of the interference term. The various amplitudes of these asymmetries have been extracted simultaneously by using the Maximum Likelihood fit method applied to the combined data taken with both charges and spin orientations of the lepton beam $[5,6]$.

Depending on the availability of collected data on a longitudinally polarized hydrogen or deuterium targets with either both charges or both helicities of the lepton beam, it is possible to extract another sets of three asymmetries by fitting the corresponding real photon leptoproduction cross 
sections. As an example, the 'incomplete' BSA amplitudes related to the interference term $\mathrm{A}_{\mathrm{L} \leftrightarrows}^{\sin (n \phi)}$, the target-spin asymmetry (TSA) $A_{U L}$ and double-spin asymmetry (DSA) $A_{L L}$ cosine amplitudes can be extracted using data from scattering of a longitudinally polarized positron beam off a longitudinally polarized deuterium target. Moreover, Using the combined data from scattering off a longitudinally polarized deuterium target with both beam charges but with only one (available) he-

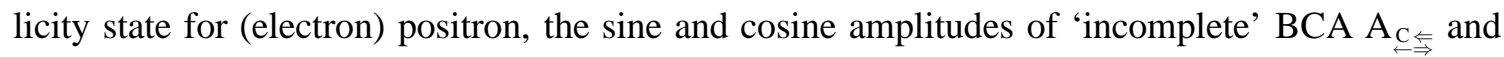
cosine amplitudes of two various DSAs, either with the 'contribution' from the BH term or without it, can be extracted.

Finally, in the case of transversely polarized hydrogen target, an additional dependence of the cross section, therefore the asymmetries, on angle $\phi_{S}$ arises, where $\phi_{S}$ is the angle between the lepton plane and the component of the target polarization vector orthogonal to the momentum of the virtual photon.

\section{Experiment and data analysis}

In order to extract these asymmetries from the data, events were selected when they contained one photon and one scattered lepton track. Photons were identified by the energy deposition in the calorimeter and preshower counter in the absence of a track. The requirements imposed on the lepton kinematics were $Q^{2}>1 \mathrm{GeV}^{2}$ and $W>3 \mathrm{GeV}$. The opening angle $\Theta_{\gamma^{*} \gamma}$ between the virtual and real photon was limited to a range of 5 to $45 \mathrm{mrad}$.

Since the recoiling nucleon (or nucleus) was not detected, exclusive DVCS events were selected imposing an additional constraint on the missing mass $M_{x}$. The exclusive region was defined as $-1.5<M_{x}<1.7 \mathrm{GeV}$ (negative $M_{x}$ values are obtained as a consequence of the finite energy resolution of the spectrometer).

\section{Results}

Single-charge BSA and BCA in hard electroproduction of photons on the hydrogen target were first reported in Refs. [8, 9] by HERMES. As an example, the results of various beam-charge asymmetry amplitudes from unpolarized [6] and polarized deuterium targets are shown in Fig. 1 as a function of $-t, x_{B}$ and $Q^{2}$ in four bins as well as for the 'overall' kinematics (left column). The errors bars (bands) represent the statistical (systematic) uncertainties. The systematic uncertainties include the contributions from the effects of acceptance, smearing, bin width and the alignment of the detectors with respect to the beam direction and are estimated from a Monte Carlo simulation. It should be noted that the contributions of coherent and associated (nucleon excites to resonances in the final states) processes could not be resolved and remain as part of the signal, while the results have been corrected for the semi-inclusive background. The hatched bands in the figure are theoretical predictions based on a model developed in Ref. [10], and calculated with a 'Regge' ansatz for modeling the $t$-dependence of the GPDs. They are calculated at the cross secion level as the sum of the incoherent processes on the proton and neutron in each kinematic bin.

A sizable magnitude of first cosine have been found for both BCA amplitudes. The data confirm that the measured $\cos (n \phi)$ amplitudes from a polarized deuterium target resemble those measured with an unpolarized one, even in the first $-t$ bin where coherent scattering contributes 


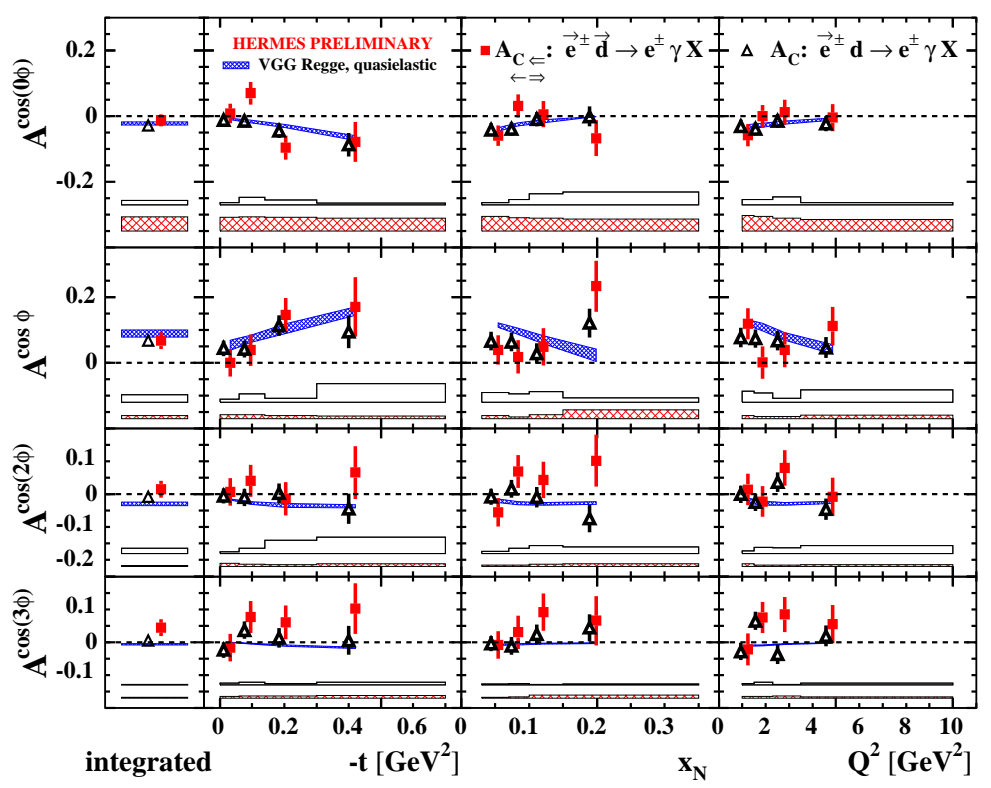

Figure 1: The BCA amplitudes for the hard exclusive electroproduction of photons from polarized and unpolarized [6] deuterium targets at the HERMES experiment. See text for details.

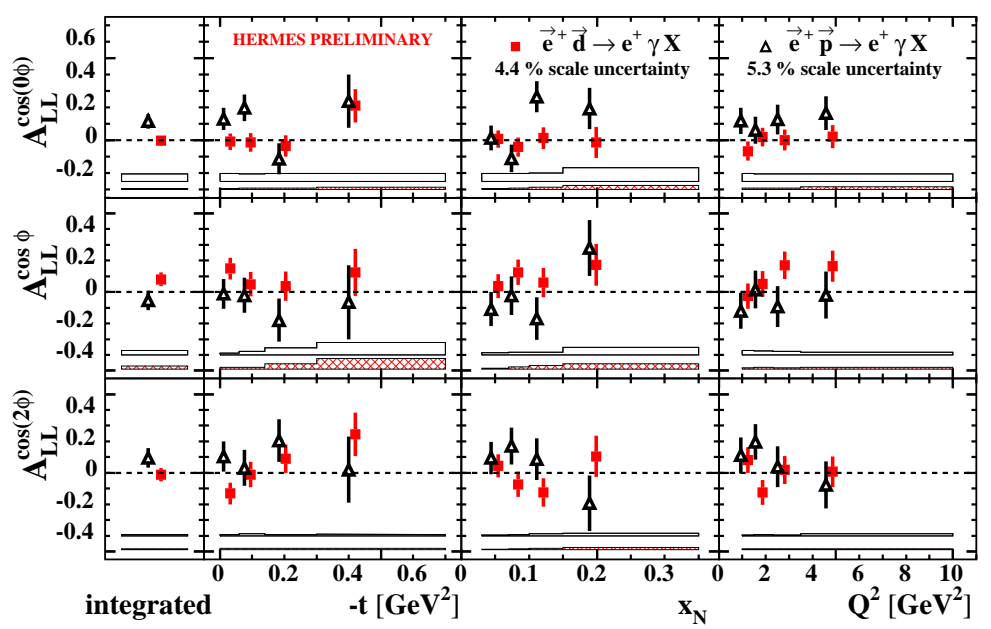

Figure 2: The DSA amplitudes $A_{\mathrm{LL}}^{\cos (n \phi)}$ for the hard exclusive electroproduction of photons from polarized hydrogen [7] and deuterium targets at the HERMES experiment. See text for details.

about $40 \%$ of the yield. This is despite the fact that $\mathrm{A}_{\mathrm{C} E}^{\cos \phi}$ for coherent scattering involves in addition the real part of the CFF $\mathrm{H}_{5}$.

The $\mathrm{A}_{\mathrm{LL}}^{\cos \phi}$ amplitude of the single-charge DSA extracted from data on polarized deuterium target and presented in Fig. 2 is slightly positive by 1.6 standard deviations of the total experimental uncertainty, while the $\mathrm{A}_{\mathrm{LL}}^{\cos (0 \phi)}$ and $\mathrm{A}_{\mathrm{LL}}^{\cos (2 \phi)}$ amplitudes are compatible with zero. Within the total experimental uncertainties these asymmetry amplitudes do not show significant differences to that measured on a longitudinally polarized hydrogen target [7], except possibly for the overall result 


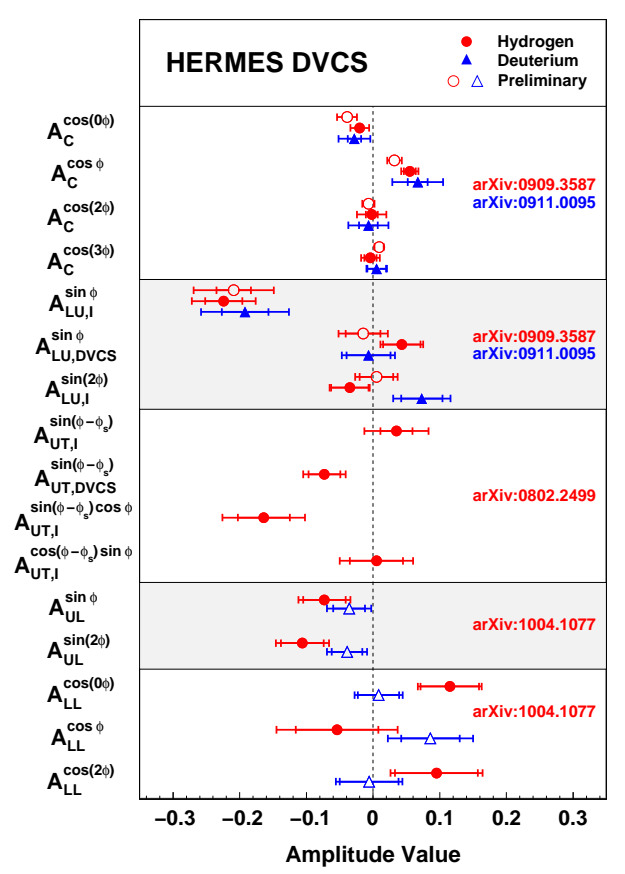

Figure 3: Overview of all DVCS azimuthal asymmetry amplitudes measured at the HERMES experiment.

for the amplitude $A_{L L}^{\cos (0 \phi)}$, where a two sigma discrepancy appears.

Figure 3 presents an overview of all azimuthal asymmetry amplitudes integarted over the entire HERMES kinematic range using the data taken in the years 1996-2007. This includes data on the unpolarized hydrogen [5] and deuterium [6] targets, the longitudinally polarized hydrogen [7] and deuterium targets and the transversely polarized hydrogen [11] target. The amplitudes are sensitive to different (combinations of) GPDs, corresponding to the real or imaginary part of the CFFs.

\section{References}

[1] D. Müller et al., Fortsch. Phys. 42, 101 (1994).

[2] X. Ji, Phys. Rev. Lett. 78, 610 (1997).

[3] A. V. Belitsky, D. Müller and A. Kirchner, Nucl. Phys. B629, 323 (2002).

[4] A. Kirchner and D. Müller, Eur. Phys. J. C32 (2004) 347.

[5] HERMES Collaboration, A. Airapetian et al., JHEP 11 (2009) 083.

[6] HERMES Collaboration, A. Airapetian et al., Nucl. Phys. B829 (2010) 1.

[7] HERMES Collaboration, A. Airapetian et al., JHEP 06 (2010) 019.

[8] HERMES Collaboration, A. Airapetian et al., Phys. Rev. Lett. 87 (2001) 182001.

[9] HERMES Collaboration, A. Airapetian et al., Phys. Rev. D75 (2007) 011103.

[10] M. Vanderhaeghen, P.A.M. Guichon and M. Guidal, Phys. Rev. D60, 094017 (1999).

[11] HERMES Collaboration, A. Airapetian et al., JHEP 06 (2008) 066. 